3.В перерабатывающей и пищевой промышленности внедрить ресурсосберегающие безотходные технологии с целью производства конкурентоспособной продукции, в том числе, сушку плодов, используя термокамеры.

4.Возобновить инфраструктуру данной отрасли.

5.Расширять формы государственной поддержки предприятий перерабатывающей и пищевой промышленности.

6.Совершенствовать межотраслевые связи в АПК РД.

7.Учитывая трудности возобновления пищевой и перерабатывающей промышленности рекомендуем Министерству сельского хозяйства РД разработать отдельное положение по субсидированию более $50 \%$ затрат по приобретению нового оборудования.

\title{
Библиографический список.
}

1. Стратегия социально-экономического развития РД до 2025 года.

2. Справочник. Сельское хозяйство Дагестана - Махачкала, 2012.

3. Государственная программа развития сельского хозяйства и регулирования рынков сельскохозяйственной продукции, сырья и продовольствия на 2013-2020 годы.

4. В. А. Кундиус. Учебное пособие. Экономика агропромышленного комплекса. Москва. Кнорус. 2011. 544. с.

5. Министерство сельского хозяйства РД. Буклет «Сельское хозяйство Дагестана» 2006 -2012 гг. Махачкала 2012.

6. Р.М. Адилов, У.Ш. Адилова. Пищевая промышленность РД Проблемы и перспективы «Проблемы развития АПК региона», №2, 2013, ДагГАУ.

\section{УДК 69.003}

Исмаилов Р.Т., Наврузбекова Н.Ф.

ПОВЫШЕНИЕ УРОВНЯ ИНТЕНСИВНОГО И ЭКСТЕНСИВНОГО ИСПОЛЬЗОВАНИЯ ОСНОВНЫХ ФОНДОВ И АНАЛИЗ ЕГО ВЛИЯНИЯ НА ОБЬЕМЫ ПРОИЗВОДСТВА

Ismailov R.T., Navruzbekova N.F.

\section{INCREASE OF LEVEL INTENSIVE AND EXTENSIVE USE OF FIXED ASSETS AND ANALYSIS OF ITS INFLUENCE ON OUTPUTS}

Рассматривается комплекс организационно-технических мероприятий, проведение которых позволяет повысить уровень интенсивного и экстенсивного использования в производственном процессе активной ча- 
сти основных фондов и таким путем увеличить объемь производства. Предлагаются методы и показатели оценки эффективности проводимых мероприятий, использование которых позволяет повысить адекватность результатов проведенного анализа и определить наиболее результативные мероприятия, обеспечивающие рост объемов производства с минимальными затратами.

Ключевые слова: строчтельное прочзводство, основные фонды, интенсивный и экстенсивный уровень использования, объемь производства, получаемая прибыль.

It is considered complex organizing-technical action, which undertaking allows to raise the level intensive and extensive of the use in production process of the active part of the main fund and such way to enlarge the volumes of production. They are offered estimations to efficiency conducted action, which use allows to raise adequacy a result called on analysis and define the most effective actions, providing growing volume of production with minimum expenses.

Key words: building production, the main funds, intensive and extensive level of the use, volumes of production, earned.

Одной из актуальных задач развития строительного производства является повышение его эффективности за счет наиболее полного использования внутрихозяйственных резервов. Для этого, прежде всего, необходимо обеспечить рациональное использование резервов основных фондов и производственных мощностей.

Увеличение объемов производства строительной продукции за счет лучшего использования основных фондов может достигаться путем:

- ввода в действие новых основных фондов и роста за счет этого производственных мощностей (при экстенсивном пути развития);

- сбалансированного использования имеющихся основных фондов с другими элементами производственного потенциала;

- эффективного использования производственных мощностей за счет задействования в производственном процессе имеющихся резервов.

К одной из главных причин, ухудшающих фондоотдачу основных фондов, является медленное их освоение при вводе в эксплуатацию. Таким образом, одной из важнейших задач повышения эффективности использования капитальных вложений и основных фондов является своевременный ввод в эксплуатацию новых основных фондов и производственных мощностей, т.е. их оперативное внедрение в производство. Сокращение сроков ввода в эксплуатацию незадействованных и новых строительных машин и механизмов позволяет быстрее получить требуемую на рынке строительную продукцию с технически более совершенных основных фондов, ускорить их оборот и тем самым, замедлить наступление морального износа основных фондов предприятия, повысить эффективность общественного производства в целом. 
Улучшение использования действующих основных фондов и производственных мощностей строительного предприятия, в том числе вновь введенных в эксплуатацию, может быть достигнуто двумя способами:

- путем повышения интенсивности использования производственных мощностей и основных фондов;

- путем повышения экстенсивности их загрузки.

Более интенсивное использование производственных мощностей и основных фондов достигается, прежде всего, за счет лучшей технической их эксплуатации и модернизации. При этом должны механизироваться и автоматизироваться не только основные производственные процессы и операции, но и вспомогательные и транспортные операции, нередко сдерживающие нормальный ход строительного производства и использование строительной техники, машин и механизмов. Устаревшие машины должны своевременно модернизироваться или заменяться новыми, более совершенными.

Интенсивность использования производственных мощностей и основных фондов повышается также путем:

- совершенствования технологий строительного производства;

- организации непрерывно-поточного производства на основании разбиения объектов на циклы с одинаковым видом строительномонтажных работ;

- выбора совместимых стройматериалов, их подготовки к производству в соответствии с требованиями заданной технологии и качества выпускаемой продукции;

- обеспечения равномерной, ритмичной работы предприятия, строительных участков и строительства отдельных объектов;

- проведения ряда других мероприятий, позволяющих повысить производительность труда и обеспечить увеличение объемов производства товарной продукции в единицу времени, на единицу мощности строительной техники.

Следовательно, интенсивный путь использования основных фондов действующих предприятий включает их техническое перевооружение и повышение доли использования в производственном процессе, а также предусматривает обновление морально и физически устаревших основных фондов. Следует заметить, что, как правило, быстрое техническое переоснащение действующих строительных предприятий особенно важно для тех из них, где имеет место значительный износ основных фондов.

Улучшение экстенсивного использования основных фондов предполагает, с одной стороны, увеличение времени работы действующей строительной техники в календарный период (в течение смены, суток, месяца, квартала, года), а с другой стороны, увеличение количества и удельного веса действующей строительной техники в составе всего имеющегося на предприятии парка строительной техники. 
Увеличение времени эксплуатации строительной техники может достигаться за счет:

- постоянного поддержания сбалансированности между производственными мощностями отдельных групп строительной техники на каждом строящемся объекте, между стройками, между отдельными предприятиями, например, внутри строительного объединения;

- путем составления расписаний, обеспечивающих эффективное перемещение строительной техники по строящимся объектам, что повышает время ее активной эксплуатации;

- улучшения технического обслуживания и ремонта основных фондов, соблюдения предусмотренных технологий производства, совершенствования организации строительного производства и труда, что способствует росту эффективности эксплуатации строительной техники, проведению ее своевременного и качественного ремонта, сокращающего простои техники в ремонте и увеличивающего межремонтный период;

- проведения мероприятий, повышающих удельный вес основных производственных операций в затратах рабочего времени, сокращения сезонности в работе предприятия, повышения сменности работы предприятия.

Известно, что на предприятиях, кроме действующей строительной техники часть ее находится в ремонте и резерве. Своевременное рациональное и эффективное использование строительной техники и механизмов за исключением части, находящейся в плановом резерве и ремонте, значительно улучшает использование основных фондов.

На успешное решение проблемы улучшения использования основных фондов, производственных мощностей и роста производительности труда оказывает значительное влияние создание крупных строительных объединений. Вместе с этим необходимо больше внимания обратить на развитие специализации производства и технического перевооружения действующих предприятий, вывод с этих предприятий несвойственной их профилю продукции, создание специализированных строительных предприятий в небольших и средних городах, где имеются резервы рабочей силы.

Важнейшим условием повышения сменности строительного производства является механизация и автоматизация производственных процессов, и в первую очередь, во вспомогательных производствах и при проведении отделочных работ, так как это позволяет перевести людей с тяжелых немеханизированных работ на квалифицированные работы во второй смене.

Ускоренные темпы механизации подъемно-транспортных, погрузочно-разгрузочных и складских работ являются основой для ликвидации имеющейся диспропорции в уровне механизации основного и вспомогательного производства на строительных предприятиях, высвобождения значительного количества вспомогательных рабочих, обеспечения попол- 
нения производственных подразделений рабочей силой, повышения коэффициента сменности работы предприятия и расширения производства без дополнительного привлечения рабочей силы. В крупных городах, имеющих дефицит рабочей силы, решение проблемы улучшения использования основных фондов и производственных мощностей действующих строительных предприятий путем их реконструкции, расширения, механизации и автоматизации производства, совершенствования организации производства и труда имеет большое значение в повышении эффективности использования их основных фондов.

Улучшение использования основных фондов и производственных мощностей зависит в значительной степени от квалификации кадров, особенно от профессиональных качеств машинистов, обслуживающих строительные машины, механизмы, агрегаты и другие виды строительной техники. Творческое и добросовестное отношение работников к труду является важным условием улучшения использования основных фондов и производственных мощностей.

Известно, что от совершенства системы морального и материального стимулирования в значительной степени так же зависит уровень использования производственных мощностей и основных фондов. Анализ техникоэкономических показателей строительных предприятий, работающих в рыночных условиях планирования и экономического стимулирования, свидетельствует, что новый экономический механизм, в том числе и введение платы за производственные фонды, пересмотр оптовых цен, применение нового показателя для определения уровня рентабельности, создание на предприятиях поощрительных фондов, способствуют улучшению использования основных производственных фондов [1].

Любой комплекс мероприятий по улучшению использования производственных мощностей и основных фондов, разрабатываемый во всех звеньях управления строительством, должен предусматривать обеспечение роста объемов производства товарной продукции. Такой рост объемов производства, в первую очередь, должен обеспечиваться за счет более полного и эффективного использования внутрихозяйственных резервов и, прежде всего, путем более полного использования машин и механизмов путем повышения коэффициента сменности, ликвидации простоев, сокращения сроков освоения вновь вводимых в действие мощностей, дальнейшей интенсификации производственных процессов.

Для проведения анализа уровня использования основных фондов обычно применяются показатели, выраженные в натуральных и стоимостных (денежных) единицах выпускаемой продукции, а также в единицах времени. Чтобы вычислить и проанализировать эффективность использования производственной мощности, применяются показатели объемов выпуска продукции в натуральном выражении.

Показатели уровня использования основных фондов, выраженные в натуральных единицах, могут быть рассчитаны по фактическим объемам 
выпуска товарной продукции, а также по максимально возможному технически расчетному ее выпуску. Однако, давая представление об общем уровне использования тех или иных строительных машин и механизмов, они не дают ответа на вопрос за счет чего получено увеличение фактической производительности строительной техники. Кроме того они не отвечают и на следующие вопросы:

- какую часть рабочего времени эта техника функционировала, а какую простаивала?;

- каков уровень интенсивности ее использования в течение этого времени?

К системе взаимосвязанных показателей непосредственно характеризующих степень экстенсивности и интенсивности использования строительной техники, машин, механизмов и производственных мощностей, а также раскрывающих резервы дальнейшего улучшения их задействования в производственном процессе, можно отнести [2]:

1) использование различного вида активной части основных фондов во времени (коэффициент их экстенсивной загрузки);

2) использование различного вида активной части основных фондов в единицу времени (коэффициент их интенсивной загрузки);

3) общее использование различного вида активной части основных фондов (коэффициент их интегральной загрузки).

Первый показатель $\left(\mathrm{K}_{\text {экст }}\right)$ определяется путем деления времени фактического использования исследуемых основных фондов на максимально возможное время их использования с учетом количества возможных смен работы предприятия. Второй показатель $\left(\mathrm{K}_{\text {инт }}\right)$ получается в результате деления фактического объема произведенной строительной продукции в единицу времени работы строительной техники, машин и механизмов, на максимально возможный объем выпуска этой продукции, который можно произвести с участием данных основных фондов в ту же единицу времени. Третий показатель (К двух показателей.

К числу показателей экстенсивного использования строительной техники, машин и механизмов на предприятии относятся также коэффициенты сменности. Они характеризует время целосменного использования активной части основных фондов, которые работают в многосменном режиме. Коэффициенты сменности рассчитывается по отдельным группам активной части основных фондов, отдельным производственным подразделениям предприятия, а также в целом по предприятию. Они показывает, сколько смен в среднем в течение суток работала строительная техника, машины и механизмы.

Показатели использования основных фондов во времени (коэффициенты экстенсивной нагрузки) определяются сравнительно просто. Показатель же использования основных фондов в единицу времени (коэффициент интенсивной нагрузки) определить для строительных предприятий можно 
также достаточно просто, если объем строительной продукции может быть выражен в натуральных единицах. Следует так же иметь в виду, что приведенные выше показатели все же не позволяют дать полного ответа на вопрос - как используются основные фонды в целом по строительному предприятию?

Роль обобщающего показателя использования основных фондов в определенной степени может выполнять показатель - объем выпуска продукции на единицу мощности строительной техники. Этот показатель выражается, как правило, в натуральных единицах.

Одним из наиболее общих показателей использования производственной мощности является коэффициент ее фактического использования, который рассчитывается путем деления объемов продукции, изготовленных за определенный промежуток времени (обычно за год), на величину производственной мощности предприятия [3]. Для предприятий, вновь введенных в эксплуатацию, обычно определяется коэффициент использования проектной мощности, представляющий собой частное от деления фактических объемов выпуска продукции на величину мощности предприятия по проекту. Этот показатель характеризует уровень освоения проектной мощности.

Следует заметить, что натуральные показатели степени использования основных фондов, также не раскрывают общей картины эффективности использования всей их совокупности строительным предприятием в целом.

Для общего анализа хозяйственной деятельности и планирования капитальных вложений, ввода в эксплуатацию основных фондов и производственных мощностей всех звеньев строительного предприятия все большее значение приобретает такой показатель эффективности производства, как выпуск продукции на 1 руб. основных фондов, который называют показателем фондоотдачи. Применяется также показатель, обратный фондоотдаче, - фондоемкость. При определении показателя фондоотдачи используются как стоимостные, так и натуральные единицы измерения.

Показатель фондоотдачи (как обобщающий стоимостный показатель использования всей совокупности основных фондов предприятия) определяется путем деления стоимости произведенной продукции на среднегодовую стоимость производственных фондов. При этом валовая продукция учитывается в неизменных ценах, а основные фонды — по полной первоначальной (или восстановительной) оценке.

Для того чтобы обойти отмеченные выше трудности использования известных показателей и получения более адекватных оценок эффективности проводимых мероприятий в работе предлагается использовать следующие показатели.

К основному показателю, отражающему эффективность проводимых предприятием мероприятий, связанных с повышением интенсивности использования строительной техники, машин и механизмов и ее влиянием на объемы выпуск строительной продукции, следует отнести прирост объема 
выпуска строительной продукции за счет повышения эффективности их использования, который может определяться согласно следующему выражению:

$$
\Delta V=\sum_{j=1}^{n}\left(\Pi_{j}^{\prime}-\Pi_{j}\right) \varphi_{j},
$$

где $\mathrm{j}$ - индекс, соответствующий j-й группе строительной техники по виду выполняемых работ (экскаваторы, подъемные краны и т.п.);

$\Pi_{j}, \Pi_{j}^{\prime}$ - производительность строительной техники ј вида до и после проведения мероприятий по повышению эффективности ее использования;

$\varphi_{j}$ - прирост объемов производства за счет роста эффективности использования строительной техники на одну условную единицу;

$n$ - количество различных групп строительной техники.

Если проводится несколько мероприятий по каждой ј группе строительной техники, то прирост чистой продукции будет определяться на основании следующего выражения:

$$
\Delta V=\sum_{j=1}^{n}\left(\sum_{i=1}^{m_{j}}\left(\Pi_{i j}^{\prime}-\Pi_{i j}\right)\right) \varphi_{j},
$$

где $\mathrm{m}_{j}$ - количество мероприятий, проводимых для повышения производительности ј группы строительной техники.

В стоимостном выражении данные показатели можно вычислить согласно следующим выражениям:

$$
\begin{gathered}
\Delta C=\sum_{j=1}^{n}\left(\Pi_{j}^{\prime}-\Pi_{j}\right) C_{j} ; \\
\Delta C=\sum_{i=1}^{n}\left(\sum_{i=1}^{m_{j}}\left(\Pi_{i j}^{\prime}-\Pi_{i j}\right)\right) C_{j},
\end{gathered}
$$

где $\mathrm{C}_{j}$ - прирост денежных средств, получаемый строительным предприятием за счет роста объемов производства на одну условную единицу.

Для определения получаемой в этом случае предприятием прибыли (П) необходимо учесть затраты, связанные с проведением мероприятий, направленных на повышение уровня использования активной части основных фондов. В этом случае получаемая после проведения мероприятий прибыль, в результате роста производительности труда, может определяться следующим образом:

$$
\Pi=\Delta C+\left(\sum_{j=1}^{n}\left(\sum_{i=1}^{m_{j}} \Psi_{i j}-\Psi_{i j}^{\prime}\right) \Pi_{\text {јобщ }}\right) \Delta \Pi_{j}-\sum_{j=1}^{n} \sum_{i=1}^{m_{j}} C_{i j},
$$

где $Ч_{i j}^{\prime}$ и $Ч_{i j}$ - численность рабочих соответственно, требующихся строительному предприятию до и после проведения і мероприятия по повышению производительности строительной техники ј группы; 


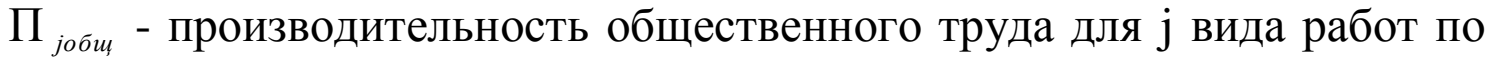
чистой продукции в базисном году;

$\Delta \Pi_{j}$ - прибыль, получаемая предприятием за счет роста производительности общественного труда для ј наименования работ;

$\mathrm{C}_{i j}$ - стоимость мероприятий i-го наименования, проводимых для $\mathrm{j}$ группы строительной техники.

Следует отметить, что если проведенные предприятием мероприятия окажутся убыточными, то приведенные выше показатели будут принимать отрицательные значения.

В заключении следует также отметить, что рассмотренные выше мероприятия позволяют повысить как интенсивность, так и экстенсивность эксплуатации активной части основных фондов, и на этой основе обеспечить рост объемов производства с учетом современного положения дел на строительных предприятиях. Предложенные методы и показатели позволяют оценивать эффективность проведения данных мероприятий и выбирать наиболее результативные из них в условиях наличия ограниченных финансовых ресурсов.

\section{Библиографический список:}

1. Черняк В.3. Экономика строительства и коммунального хозяйства. М.: ЮНИТИ-ДАНА, 2003.

2. Савицкая Г.В. Анализ хозяйственной деятельности предприятия. - М.: ИНФРА - М, 2000.

3. Савельев А.А. Методы управления потенциалом промышленного предприятия. - СПб.: Политехника, 2006. 